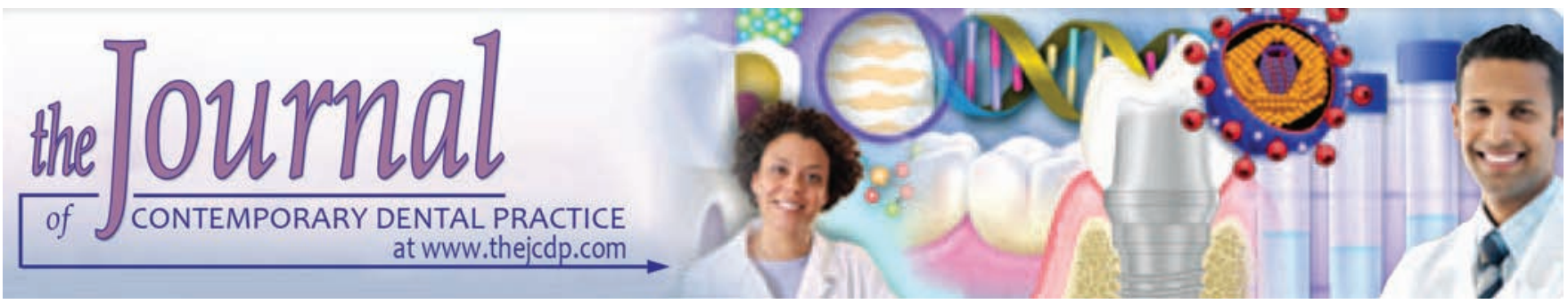

\title{
Swinging Replantation: A Possible Protocol for the Management of Inverted Impacted Upper Central Incisor Tooth
}

\author{
${ }^{1}$ Seidu A Bello, ${ }^{2}$ Deborah J Enebong, ${ }^{3}$ Emeka V Obi
}

\begin{abstract}
Introduction: Inverted tooth impaction is a rare occurrence. Third molar impaction is the most extensively studied, and only eight cases of inverted third molars have been reported in a literature search of 40 years from 1973 to 2013. In a study, 43.4\% of the premaxillary supernumeraries were inverted and $21.1 \%$ were transversely oriented, while occasional inverted central incisors have been mentioned in the literature. Severe dilaceration makes the management of this case a big challenge. The case of an 8-year-old boy with an inverted, rotated, and impacted upper left central incisor obstructed by an odontome is presented here. Under local anesthesia, the odontome was excised; the inverted tooth was swung and repositioned. The patient experienced uneventful healing, and with 30 months follow-up, progressive tooth eruption has been observed. Vitality test is positive, while radiologically there was no sign of resorption. Swinging replantation is a protocol that has been used for the management of an inverted, rotated, and impacted central incisor. Open root apex is an important factor in the selection of the method. The minimal periodontal damage prevents inflammatory and replacement resorption. With some clear advantages over options like orthodontic and prosthetic replacement, it could be a method to be considered in the management of severely dilacerated central incisors.

The clinical significance is that even though management of a severely malpositioned tooth has always been a big challenge in dental practice, requiring extraction and prosthodontic replacement, swinging replantation provides a possible protocol of tooth retention, i.e., less expensive and associated with fewer hospital visits.
\end{abstract}

Keywords: Inverted and impacted, Swinging replantation, Upper central incisor.

How to cite this article: Bello SA, Enebong DJ, Obi EV. Swinging Replantation: A Possible Protocol for the Management

\footnotetext{
${ }^{1-3}$ Department of Dental and Maxillofacial Surgery, State House Medical Centre, Abuja, Nigeria
}

Corresponding Author: Seidu A Bello, Department of Dental and Maxillofacial Surgery, State House Medical Centre, Abuja Nigeria, Phone: +2348054929034, e-mail: sabello2004@yahoo. com, seidubello@gmail.com of Inverted Impacted Upper Central Incisor Tooth. J Contemp Dent Pract 2017;18(1):69-73.

Source of support: Nil

Conflict of interest: None

\section{INTRODUCTION}

Inverted tooth impaction is a rare occurrence. ${ }^{1}$ Dilaceration is a comparatively uncommon dental deformity generally characterized by an angulation between crown and root, and consequently causing noneruption of the tooth. ${ }^{2}$ Tooth impaction is a condition in which a tooth fails to erupt into normal position beyond the expected time. ${ }^{3}$ Commonly, tooth impaction occurs in permanent dentition and rarely happens in primary teeth. Most of the primary teeth impactions are seen in second molars. ${ }^{4,5}$ Any permanent tooth in the dental arch can be impacted, but the teeth most frequently involved in a descending order are the mandibular and maxillary third molar, the maxillary canines, the mandibular and maxillary second premolar, and maxillary central incisors. ${ }^{6}$ The many kinds of impaction include vertical, horizontal, buccal, lingual, and inverted. ${ }^{1}$ Third molar impaction is the most extensively studied and only eight cases of inverted third molars have been reported in a literature search of 40 years from 1973 to $2013 .{ }^{1}$ In another study, $43.4 \%$ of the premaxillary supernumeraries were inverted and $21.1 \%$ were transversely oriented. ${ }^{7}$ Occasional inverted central incisors have been mentioned in the literature. ${ }^{2}$

Management of inverted impacted central incisors includes various multidisciplinary approaches varying from space creation to prosthodontic rehabilitation and reimplantation to endodontic treatment. ${ }^{8}$ The impacted dilacerated incisor has been successfully treated with the aid of orthodontic traction. ${ }^{2}$ Removal of any of the local obstructions to an unerupted tooth is an important step in the management of such a tooth. Extraction and 
replantation is one of the possible protocols of management, but reports are scanty in the literatures. Replantation of a tooth after a traumatic avulsion is quite popular either followed with or without root canal treatment. ${ }^{9}$ Intentional replantation is another relatively popular terminology in endodontic treatment. ${ }^{10}$ It involves the atraumatic extraction of the offending tooth, root-end resection/preparation/filling, and reinsertion of the extracted tooth. ${ }^{10,11}$

This study presents an inverted impacted and rotated central incisor obstructed by a complex odontome. It was successfully treated by excision of the odontome followed by a novel method of swinging replantation. This is being contributed to the body of knowledge as a possible protocol in the management of such conditions.

\section{CASE REPORT}

An 8-year-old boy presented at the Oral and Maxillofacial Surgery Unit of State House Medical Centre in September 2012 with a complaint of unerupted tooth no. 21, while the contralateral tooth no. 11 was fully erupted. There was no

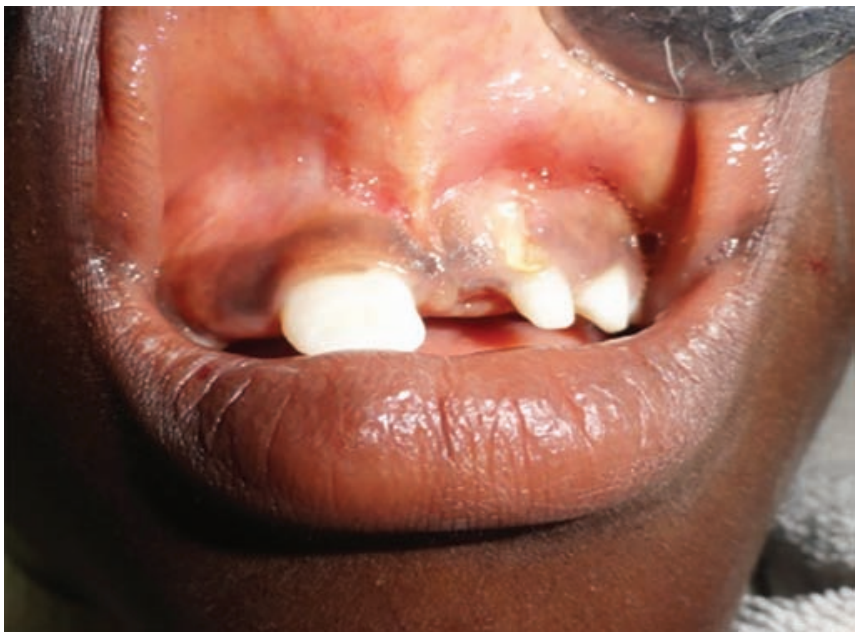

Fig. 1: Intraoral examination at presentation showing a missing 21 and fully erupted 11

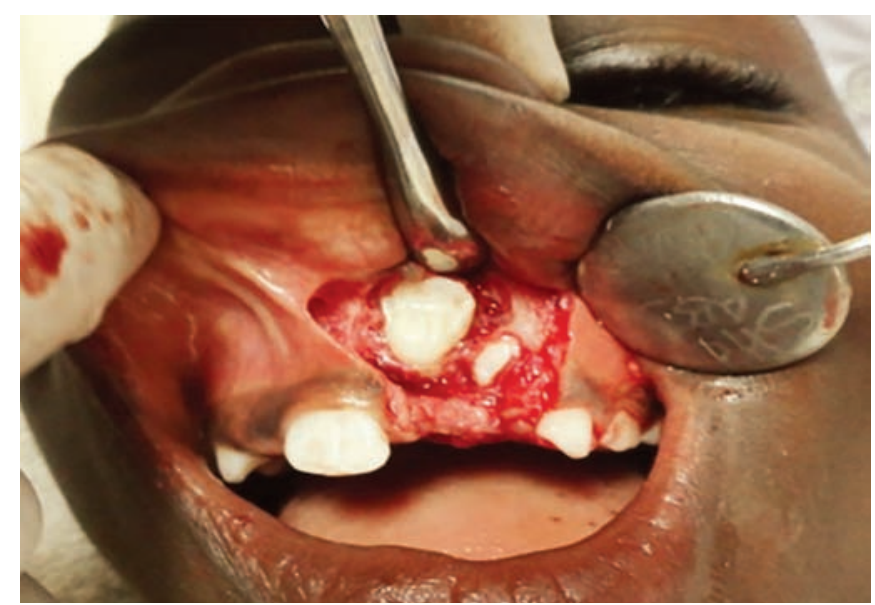

Fig. 3: Inverted and rotated left central incisor was properly exposed history of trauma by the informant (mother). Examination revealed a young boy not in an obvious painful distress. He was systemically stable. Intraoral examination revealed a missing 21 and fully erupted 11 (Fig. 1).

Radiological examination shows inverted 21 with an opaque mass incisal to the tooth. There was no sign of a cyst surrounding the tooth (Fig. 2). The contralateral 11 was fully erupted with open apices. A diagnosis of inverted impacted left central incisor obstructed by a complex odontome was made.

Under local anesthesia, a mucoperiosteal flap was raised to expose the odontome. The odontome was curetted with a periosteal elevator, thereby exposing the inverted and $180^{\circ}$ rotated upper left central incisor (Fig. 3). The tooth was swung gently with a periosteal elevator over $180^{\circ}$ bringing to a completely derotated position. The tooth was flexibly splinted to the central incisor using a 0-vicryl resorbable suture. The flap was replaced and closed with remaining suture 0 -vicryl round the tooth for further splinting (Fig. 4). The patient was placed on amoxicillin

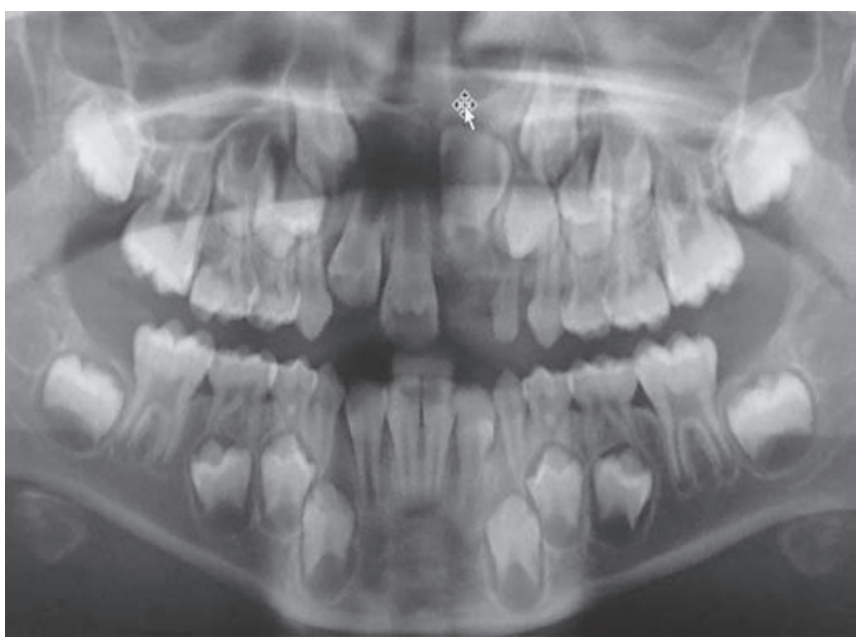

Fig. 2: A panoramic view of the patient showing inverted 21 with an opaque mass incisal to the tooth. Contralateral tooth no. 11 is fully erupted

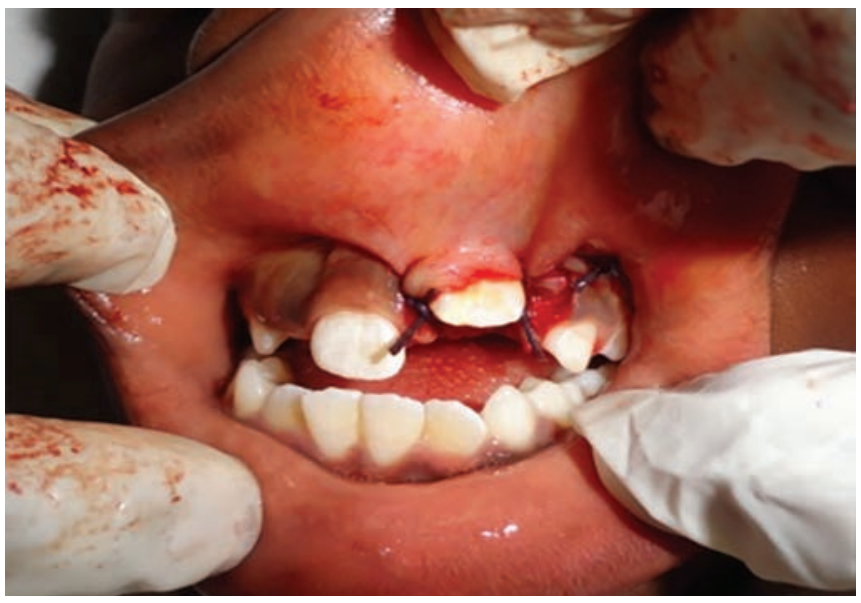

Fig. 4: Tooth no. 21 was swung over $180^{\circ}$ and flexibly splinted with resorbable suture 


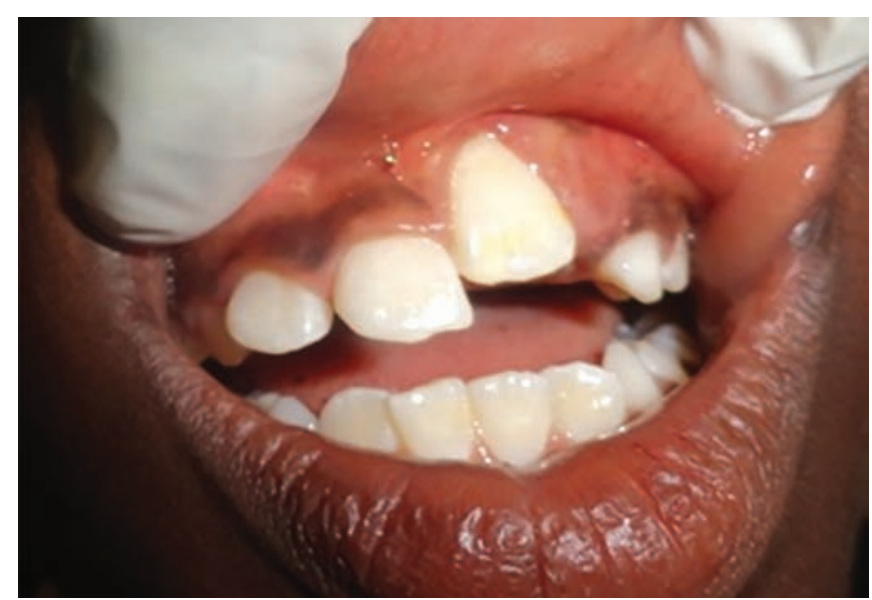

Fig. 5: Fifteen months follow-up, progressively erupting 21, which is about $5 \mathrm{~mm}$ short of incisal plane

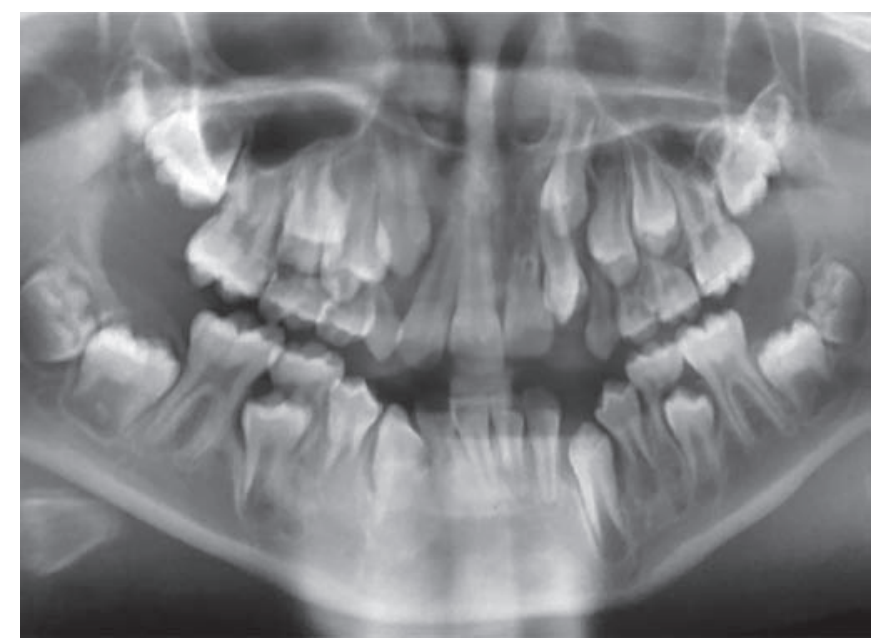

Fig. 7: Panoramic view at 27 months showing almost fully erupted tooth root with no sign of ankyloses

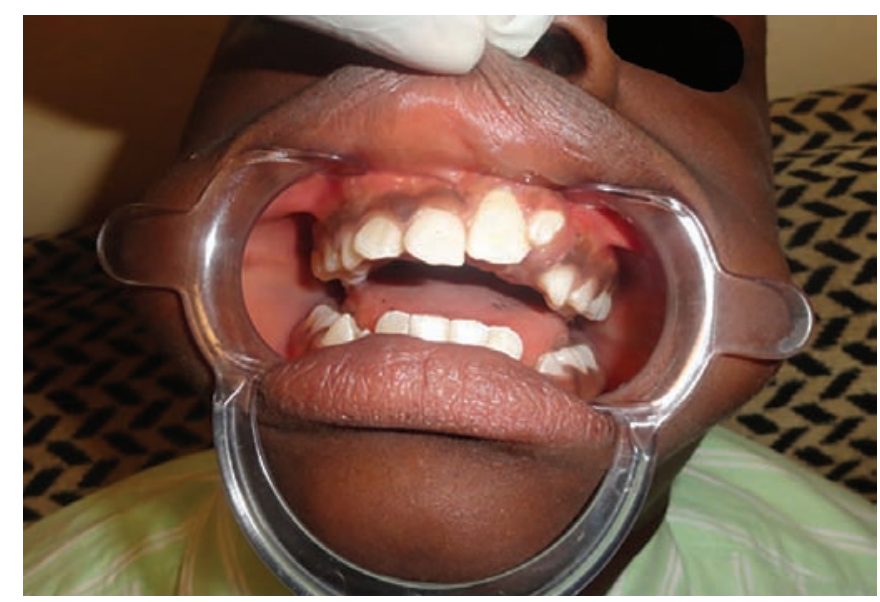

Fig. 6: Twenty-seven months follow-up, progressively erupting 21 , which is about $2 \mathrm{~mm}$ short of incisal plane

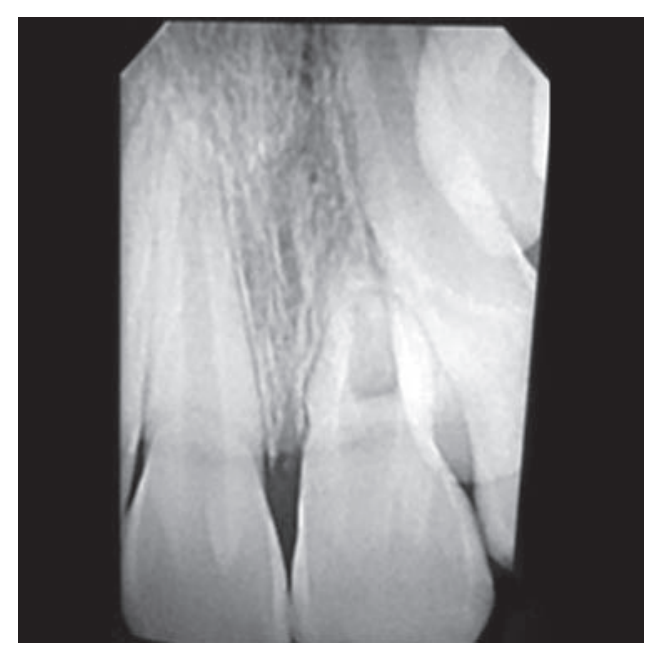

Fig. 8: A periapical X-ray taken at 27 months, showing fully developed (closed root apex) tooth no. 21

and Gardner's syndrome. ${ }^{12}$ The case under discussion was due to a complex odontome, which is an opaque mass without resemblance with a tooth structure. This is in contrast with a compound odontome that resembles a tooth but usually smaller than corresponding tooth.

Removal of obstruction is the first step in the management of an impacted tooth due to an obstacle. Thereafter the choice of method of alignment of the tooth will be greatly affected by position of the tooth in the arch, the orientation of the tooth to the incisal plane, available expertise, and the level of root development of the tooth. Whether the tooth follicle is associated with pathology or is pathology-free could also influence the choice of management. ${ }^{8}$ An impacted tooth i.e., lesser than $3 \mathrm{~mm}$ of incisal plane and well aligned along the contralateral tooth has the chance of spontaneous eruption following removal of an obstacle. A more superiorly placed tooth or tooth with mild angulation could be aligned with the aid of orthodontic traction. An inverted tooth with $180^{\circ}$ orientation could be a real challenge in orthodontic factors include alveolar cleft, cleidocranial dysplasia, 
consideration. ${ }^{2}$ In such circumstances, extraction with prosthodontic (implant or bridge) replacement or tooth replantation could be considered.

When the tooth is removed from its socket consequence of a trauma, and the surrounding structures of periodontal ligament and neurovascular bundle injured, the situation is regarded as "tooth avulsion" in the World Health Organization's classification system modified by Andreasen. ${ }^{13}$ Extraction of a tooth is similar to tooth avulsion; in the sense that the surrounding structures become injured. As it is obtainable with tooth avulsion where successful replantation has been carried out, so it is with extraction and replantation.

Swinging replantation is a form of incomplete extraction. Following extraction or avulsion, the blood supply to the tooth is disrupted. The healthy cell survival rate of pulp and periodontal ligament fibers begins to decrease by lack of blood supply, dryness, and possible bacterial contamination. ${ }^{14}$ In case of damage to protective structures of the root surface (i.e., cementum, periodontal ligament), a chemotactic process will attract clastic cells to site of injury, initiating the resorption process. External inflammatory resorption is a progressive inflammatory process and a necrotic pulp can induce and sustain this resorptive destruction. ${ }^{15}$ In the case under consideration, swinging of the tooth, being an incomplete extraction process, may be associated with mild or no disruption of pulpal blood supply, limits the destruction to the protective surface of the tooth, thereby limiting the process of inflammation and ensuring successful healing and progressive eruption of the tooth.

Innermost cell layers of the root surface of a tooth should be vital for the proper regeneration of the periodontal ligament. Although $\mathrm{pH}$, osmolality, and temperature of the storage medium are important for the survival of periodontal ligament cells, wet storage is the main environment to save the avulsed tooth. ${ }^{16,17}$ In swinging replantation as presented, the periodontal surface is constantly kept wet and hence, the higher chance of cell survival and tooth development. Another important factor is the open apices of the root of the tooth under consideration. This ensures adequate blood supply and greater chance of tooth development.

The other type of resorption which occurs due to lack of the vital periodontal ligament of a tooth is called replacement resorption (ankylosis). ${ }^{15}$ In this type of resorption, while osteoclastic activity is resorbing the root surface, osteoblastic activity creates the new bone. Clinically, the tooth is immobile and presents a metallic sound to percussion. Infraoccluded teeth are the common observation. There may be no radiographic signs. ${ }^{18}$ Open tooth root apex coupled with minimal periodontal injury caused by incomplete extraction reduces or totally eliminated the possibility of replacement resorption in the case under consideration, where progressive root development was observed.

Swinging replantation is a method of choice in situation where orthodontics is not feasible due to unfavorable tooth angulation, lack of funds, or necessary expertise. It is also associated with reduced hospital visit. When compared with extraction and prosthetic replacement, it is a treatment that could be carried out at a younger age whereas prosthetic replacement has an age consideration. Since progressive root growth was noticed and no replacement resorption, minor imperfections could be corrected by general orthodontic treatment at a later stage.

\section{CONCLUSION}

Swinging replantation is a protocol that has been successfully employed for the management of an inverted, impacted, and rotated central incisor. Open root apex is an important factor in the selection of the method. The minimal periodontal damage prevents inflammatory and replacement resorption. With some clear advantages over options like orthodontic and prosthetic replacement, it could be a method to be considered in the management of severely malpositioned central incisors.

\section{CLINICAL SIGNIFICANCE}

Management of a severely malpositioned tooth has always been a big challenge in dental practice, requiring long period of treatment including extraction and prosthodontic replacement. Swinging replantation provides a possible protocol of tooth retention, i.e., less expensive and associated with fewer hospital visits.

\section{REFERENCES}

1. Togoo RA. Rare occurrence of inverted maxillary third molar impaction: a case report. J Int Oral Health 2013 Jun;5(3):85-87.

2. Xue JJ, Ye NS, Li JY, Lai WL. Management of an impacted maxillary central incisor with dilacerated root. Saudi Med J 2013 Oct;34(10):1073-1079.

3. Lambert M, Rothman DL. Unusual impaction of a primary lateral incisor. ASDC J Dent Child 1994 Mar-Apr;61(2):146-148.

4. Albers DD. Ankylosis of teeth in developing dentition. Quintessence Int 1986 May;17(5):303-308.

5. Bianchi SD, Roccuzzo M. Primary impaction of primary teeth: a review and report of three cases. J Clin Pediatr Dent 1991 Spring;15(3):165-168.

6. Al-Faleh W. Completely Impacted teeth in dentate and edentulous jaws. Pak Oral Dent J 2009;29(2):255-260.

7. Liu D, Zhang W, Zhang Z, Wu Y, Ma X. Three-dimensional evaluations of supernumerary teeth using cone-beam computed tomography for 487 cases. Oral Surg Oral Med Oral Pathol Oral Radiol Endod 2007 Mar;103(3):403-411.

8. Mohan S, Kankariya H, Fauzdar S. Impacted inverted teeth with their possible treatment protocols. J Maxillofac Oral Surg 2012 Dec;11(4):455-457.

9. Tezel H, Atalayin C, Kayrak G. Replantation after traumatic avulsion. Eur J Dent 2013 Apr-Jun;7(2):229-232. 
10. Asgary S, Alim Marvasti L, Kolahdouzan A. Indications and case series of intentional replantation of teeth. Iran Endod J 2014 Winter;9(1):71-78.

11. Bender I, Rossman L. Intentional replantation of endodontically treated teeth. Oral Surg Oral Med Oral Pathol 1993 Nov;76(5):623-630.

12. Seraj B, Ghadimi S, Mighani G, Zare H, Rabbani M. Inverted impacted primary maxillary incisors: a case report. J Dent (Tehran) 2012 Spring;9(2):174-177.

13. Andreasen JO, Andreasen FM. Classification, etiology and epidemiology of traumatic dental injuries. In: Textbook and Color Atlas of traumatic injuries of the teeth. Copenhagen: Munksgaard; 1994. p. 151-177.

14. Adil NF, Ahmed SS, Jindal MK, Arshad SH. Delayed replantation of avulsed teeth. J Indian Soc Pedod Prev Dent 2007;(Suppl 25)S17-S19.
15. Moradian H, Badakhsh S, Rahimi M, Hekmatfar S. Replantation of an avulsed maxillary incisor after 12 hours: three-year follow-up. Iran Endod J 2013 Winter;8(1):33-36.

16. Andreasen JO, Borum MK, Jacobsen HL, Andreasen FM. Replantation of 400 avulsed permanent incisors 4 factors related to periodontal ligament healing. Endod Dent Traumatol 1995 Apr;11(2):76-89.

17. SigalasE, ReganJD,KramerPR, WitherspoonDE, Opperman LA. Survival of human periodontal ligament cells in media proposed for transport of avulsed teeth. Dent Traumatol 2004 Feb;20(1):21-28.

18. de Aguiar Santos BO, de Mendonça DS, de Sousa DL, Neto JJSM, de Araújo RBR. Root resorption after dental traumas: classification and clinical, radiographic and histologic aspects. RSBO 2011 Oct-Dec;8(4):439-445. 